\title{
Duelo anticipado en familiares de personas con enfermedad de flzheimer: análisis del discurso
}

\section{finticipated Grief in Relatives of People with fllzheimer’s Disease: Discourse finalysis Duelo antecipado em parentes de pessoas com doença de flzheimer: análise de conteúdo}

\author{
Karen Juan Calabuig \\ Laura Lacomba-Trejo \\ Marián Pérez-Marín \\ Departamento de Personalidad, Evaluación y Tratamientos Psicológicos, Universitat de València
}

Doi: https://doi.org/10.12804/revistas.urosario.edu.co/apl/a.8436

\section{Resumen}

La enfermedad de Alzheimer (EA) es la demencia más frecuente, considerada un proceso crónico e incurable. Los cuidadores de personas con EA pueden experimentar duelo anticipado. El objetivo fue observar el desarrollo de duelo anticipado en familiares de personas con EA. Participaron 10 cuidadores familiares de personas con EA en estado moderado o avanzado (70\% mujeres), de entre 18 y 80 años. Respondieron una entrevista semiestructurada (sentimientos, reconocimiento de la muerte, reorganización familiar, esperanza, facilitación o resistencia a la muerte y aproximación o distanciamiento del familiar). Se em- pleó la técnica del análisis cualitativo del contenido empleando la triangulación de dos investigadoras. Además, se realizó un análisis descriptivo con SPSS v.26. Los resultados sugieren que los familiares de personas con EA pasan por un proceso de duelo anticipado: muestran sorpresa, ira y miedo; la mayoría (90\%) reconocía que su familiar estaba al final de la vida; todos realizaron reestructuraciones familiares para adaptarse a la nueva situación; el 30\% tenía la esperanza de que la salud de su familiar mejorara, mientras que el $70 \%$ facilitaría el proceso de muerte. Así mismo, la mayoría se acercó más a su familiar $(80 \%)$ y la mitad $(50 \%)$ cambió positivamente

Karen Juan Calabuig ORCID ID: https://orcid.org/0000-0003-0296-3276

Laura Lacomba-Trejo ORCID ID: https://orcid.org/0000-0002-1990-9711

Marián Pérez-Marín ORCID ID: https://orcid.org/0000-0003-3532-8818

Dirigir correspondencia a Marián Pérez-Marín. Dirección: Av. Blasco Ibáñez, 21, 46010, Departamento de Personalidad, Evaluación y Tratamientos Psicológicos, Facultat de Psicologia i Logopedia, Universitat de València, Valencia, España. Teléfono: + 34963864100. Correo electrónico: marian.perez@uv.es

Las autoras quieren mostrar su agradecimiento a los cuidadores familiares que participaron en la investigación compartiendo sus experiencias.

Para citar este artículo: Calabuig, K., Lacomba-Trejo, L., \& Pérez-Marín, M. (2021). Duelo anticipado en familiares de personas con enfermedad de Alzheimer: análisis del discurso. Avances en Psicología Latinoamericana, 39(2), 1-17. https://doi.org/10.12804/ revistas.urosario.edu.co/apl/a. 8436 
su actitud hacia la muerte. Se identificaron mayores dificultades en mujeres, cuidadores principales convivientes con EA y que tienen un nivel de estudios básicos. Conocer los factores de riesgo y protección en el duelo anticipado puede ayudar a detectar a las personas en riesgo, pudiéndose intervenir psicológicamente, potenciando los factores de protección.

Palabras clave: duelo anticipado; cuidadores familiares; enfermedad de Alzheimer; estudio mixto.

\section{fibstract}

Alzheimer's disease (AD) is the most common dementia; it is considered a chronic and incurable process. Caregivers of people with AD may experience anticipatory grief. The aim was to observe the development of anticipatory grief in family caregivers of people with AD. Ten family caregivers of people with moderate or advanced AD (70\% women), aged between 18 and 80 years, participated. They answered a semi-structured interview (feelings, recognition of death, family reorganisation, hope, facilitation or resistance to death and approaching or distancing from the relative). The technique of qualitative content analysis was employed using the triangulation of two researchers. In addition, a descriptive analysis was carried out with SPSS V.26. The results suggest that relatives of people with AD go through a process of anticipatory grief: they show surprise, anger and fear; the majority (90\%) recognised that their relative was at the end of life; all engaged in family restructurings to adapt to the new situation; $30 \%$ were hopeful that their relative's health would improve, while $70 \%$ would facilitate the dying process. In addition, most of them became closer to their relative $(80 \%)$ and half of them $(50 \%)$ changed their attitude towards death in a positive way. Greater difficulties were identified in: women, main caregivers living with AD and those with a basic level of education. Knowing the risk and protective factors in anticipatory bereavement can help to detect people at risk and to intervene psychologically by strengthening the protective factors.

Keywords: Anticipated grief; family caregivers; Alzheimer's disease; mixed study;

\section{Resumo}

A doença de Alzheimer (AD) é a demência mais comum; é considerada um processo crónico e incurável. Os prestadores de cuidados de pessoas com AD podem experimentar um luto antecipado. O objectivo era observar o desenvolvimento do luto antecipado nos cuidadores familiares das pessoas com AD. Dez cuidadores familiares de pessoas com DA moderada ou avançada (70\% mulheres), com idades compreendidas entre os $18 \mathrm{e}$ os 80 anos, participaram. Responderam a uma entrevista semi-estruturada (sentimentos, reconhecimento da morte, reorganização familiar, esperança, facilitação ou resistência à morte e aproximação ou afastamento do parente). A técnica de análise qualitativa do conteúdo foi utilizada utilizando a triangulação de dois investigadores. Além disso, foi realizada uma análise descritiva com o SPSS v.26. Os resultados sugerem que os familiares das pessoas com DA passam por um processo de luto antecipado: mostram surpresa, raiva e medo; a maioria (90\%) reconheceu que o seu familiar estava no fim da vida; todos fizeram reestruturações familiares para se adaptarem à nova situação; $30 \%$ esperavam que a saúde do seu familiar melhorasse, enquanto $70 \%$ facilitariam o processo de morte. Além disso, a maioria deles aproximou-se dos seus parentes ( $80 \%)$ e metade deles (50\%) mudou a sua atitude em relação à morte de uma forma positiva. Foram identificadas maiores dificuldades em: mulheres, principais cuidadoras que vivem com AD e aquelas com um nível básico de educação. Conhecer os factores de risco e de protecção em luto antecipado pode ajudar a detectar pessoas em risco e a intervir psicologicamente, reforçando os factores de protecção.

Palavras-chave: luto antecipado; cuidadores familiares; doença de Alzheimer; estudo misto.

El Alzheimer (EA) es una enfermedad crónica neurodegenerativa de origen multifactorial y evolución progresiva que se caracteriza clínicamente por un deterioro cognitivo continuo, y neuropatológicamente por la presencia de ovillos neurofibrilares y placas seniles (Marín-Carrasco, 2009). 
A pesar de que se estima la forma más frecuente de demencia (el 60-70\% de los casos) (Organización Mundial de la Salud [OMs], 2019), hasta hoy, se considera incurable (Marín-Carrasco, 2009). La EA es un problema de salud pública, puesto que continúa aumentado y los costes personales, sociales y económicos asociados a ella son elevados (Aranda \& Calabria, 2019).

La EA evoluciona en forma de fases (leve, moderada y avance), observándose que en fase avanzada las personas suelen presentar un gran nivel de dependencia caracterizado por graves dificultades para reconocer a sus familiares o a sí mismos, mutismo, pérdida del control de esfínteres y de la movilidad, y en algunos casos, posibilidad de requerir alimentación asistida (Alzheimer's Association [AA], 2018). Como enfermedad crónica, requiere de grandes periodos de cura y tratamiento paliativo, lo que implica un fuerte impacto psicológico personal, familiar y social (Casal et al., 2019).

Cuidar de una persona en situación de dependencia, como es el caso de la EA en estadios más avanzados, es un acontecimiento familiar de vital importancia que puede generar sobrecarga emocional (Hernández et al., 2019). La familia se ve implicada en un proceso de ajuste para lograr un nuevo equilibrio a medida que las necesidades de la persona con EA cambian. De este modo, la familia necesita cubrir los roles que antes ocupaba la persona con EA; aun así, es probable que diferentes miembros de la familia asuman de diversa manera la pérdida parcial de su familiar y tengan distintos grados de éxito en la aceptación y adaptación de la nueva situación (Crespo et al., 2013).

Amedida que avanza la enfermedad, la atención directa e indirecta de la persona con EA, se hace cada vez más difícil. Esta tarea puede requerir una gran cantidad de tiempo y de esfuerzo, repercutiendo de forma potencialmente negativa en la salud física y emocional de los familiares (Hernández et al., 2019; Ruiz-Fernández et al., 2019). De hecho, la mayoría de estos familiares están en una posición tanto de prestar atención como de recibirla, motivo por el que se ha clasificado a los cuidadores como "los pacientes ocultos" (Wilkinson \& Lynn, 2005). Así, resulta frecuente que desarrollen síndrome de burnout y sintomatología emocional de relevancia (Palacios-Espinosa \& González, 2015; Rodríguez et al., 2019; Viñas-Diez et al., 2019). Hay que tener en cuenta que la sintomatología emocional del cuidador principal puede facilitar el desajuste físico y emocional de la persona al final de la vida (Soto-Rubio et al., 2018; Hernández et al., 2019), $\mathrm{y}$ es un predictor de la claudicación del cuidado (Zepeda-Álvarez \& Muñoz-Mendoza, 2019).

Entonces, el continuo deterioro de la persona con EA, los cambios de roles y adaptaciones familiares, hacen más posible que el cuidador pariente empiece a desarrollar un proceso de duelo anticipado (Vilajoana, 2017). El duelo anticipado se define como un fenómeno que puede ocurrir cuando la persona tiene la posibilidad de anticipar la muerte de un ser querido o la propia, permitiendo al individuo comprender la pérdida como un proceso natural y desarrollar estrategias de afrontamiento para que sea menos dolorosa la pérdida (Rando, 2000).

Cuando una persona sufre un duelo anticipado se pueden observar sentimientos inherentes al duelo y a la aceptación de las pérdidas; reorganización individual y familiar; anticipación de los reajustes necesarios; desinterés; facilitación de una muerte apropiada (Lindemann, 1994; Rando, 2000); reconocimiento inevitable de la muerte; reconciliación y desarrollo de una perspectiva que facilite la creencia de que la vida es valiosa (Futterman et al., 1972) y posibilidad de aparición de esperanza (Kübler-Ross, 1998). Es decir, no solo la muerte de la persona con EA puede generar un duelo, sino que la pérdida asociada a las capacidades físicas y cognitivas puede hacer que se dé un duelo anticipado (Pérez-Godoy \& Moreno-Guerín, 2007).

El duelo anticipado se ha señalado por algunos autores como un factor protector, puesto que puede ayudar a paliar los síntomas depresivos una vez el familiar ha fallecido (Parkes \& Weiss, 1983; Stroebe et al., 2001). Otros autores mantienen que 
es un factor de riesgo, debido a que en el duelo anticipado se da una amnesia emocional temporal que después desencadena en sintomatología más grave (por ejemplo, trastornos ansiosos, trastornos depresivos, trastornos relacionados con el estrés y duelo complicado) (Gatto, 2006; Loitegui, 2008). No obstante, los datos son controvertidos, algunos estudios no lo señalan como factor relevante para el desarrollo posterior de duelo patológico (Yi, 2015). Por ello, hemos de tener en cuenta que el duelo anticipado es un proceso normativo ante una pérdida (Castillo et al., 2019) que permite al cuidador familiar prepararse para la despedida, poder compartir sus sentimientos con su ser querido al final de la vida y ajustarse a la nueva situación (Meza et al., 2008).

Aunque en los últimos años ha habido un aumento de la literatura que apoyó la postura de duelo anticipado como factor de riesgo, realmente no existe una evidencia clara que nos permita afirmarlo. Por eso, el presente estudio tiene el objetivo general de estudiar el desarrollo del duelo anticipado en familiares de personas con EA. Concretamente, (1) conocer la presencia de características de duelo anticipado en cuidadores de personas con EA avanzado y (2) analizar la aparición de las dimensiones del duelo anticipado en función de las variables sociodemográficas estudiadas (sexo, edad, situación laboral, situación académica, religión, cuidador familiar principal y convivencia).

\section{Método}

\section{Participantes}

Los participantes fueron 10 familiares $(70 \%$ mujeres) de personas con EA en fase moderada-grave, con relación afectiva próxima (entendiendo por próxima que tuvieron contacto regular con la persona con EA). Los criterios de exclusión fueron sufrir cualquier tipo de discapacidad mental y pertenecer a edades extremas (menos de 18 o más de 80 años).

\section{Materiales e instrumentos}

Se realizaron registros ad hoc para recoger información en relación con las variables sociodemográficas y médicas de interés del cuidador familiar y de la persona con EA. En ese sentido, de los cuidadores familiares se tuvo en cuenta: el sexo, la edad, el nivel de estudios, el nivel socioeconómico y el grado de religiosidad (en función de las siguientes categorías: creyente y practicante, creyente y no practicante, y no creyente, no practicante), la convivencia o no con la persona con EA, el grado de percepción de vínculo afectivo, los cargos familiares y el apoyo social. Por su parte, de la persona con EA se tuvo en cuenta la edad y el grado de afectación de la EA.

Además, se creó una entrevista semiestructurada de 16 preguntas para llevar a cabo la presente investigación. La entrevista fue elaborada teniendo en cuenta las diferentes dimensiones propuestas en la literatura alrededor del duelo anticipado. Por lo tanto, las variables fueron: sentimientos, reconocimiento de la muerte, reorganización familiar, esperanza, facilitación o resistencia a la muerte y aproximación o distanciamiento del familiar(Bowlby, 1983; Freud, 1917; Futterman et al., 1972; Gatto, 2006; Kübler-Ross, 1998; Lindemann, 1994; Rando, 2000). De la misma manera, se incluyó como dimensión la opinión de la persona en relación con la muerte. El contenido de las dimensiones de la entrevista se puede consultar en la tabla 1.

\section{Procedimiento}

Previa evaluación y firma del consentimiento informado, se explicó el objetivo de la investigación a los participantes, los posibles riesgos que podía tener la entrevista y los beneficios sociales que podían derivarse de su participación. Después de seleccionar a los participantes teniendo en cuenta los criterios de inclusión y exclusión, firmaron el consentimiento informado tanto para ser evaluados como para ser grabados en audio, además de 
Tabla 1.

Dimensiones de la entrevista

Contenido

\begin{tabular}{ll}
\hline Emociones y sentimientos & $\begin{array}{l}\text { Emociones y sentimientos experimentados por el cuidador familiar ante el diagnóstico, } \\
\text { en el proceso de la EA y al estar con su familiar. }\end{array}$ \\
\hline Reconocimiento de muerte & $\begin{array}{l}\text { Asimilación de la posibilidad de muerte de la persona con EA y presencia de } \\
\text { sentimientos de pérdida. }\end{array}$ \\
\hline Reorganización familiar & $\begin{array}{l}\text { Cambio en los roles familiares debido a la presencia de enfermedad en un miembro de } \\
\text { la familia. }\end{array}$ \\
\hline Esperanza & Sentimientos de esperanza de que el familiar se recupere y no fallezca. \\
\hline $\begin{array}{l}\text { Facilitación o resistencia a } \\
\text { la muerte }\end{array}$ & $\begin{array}{l}\text { Reacción ante la etapa de final de la vida. ¿La persona facilita la situación de muerte } \\
\text { digna o prefiere la asistencia médica continuada? }\end{array}$ \\
\hline $\begin{array}{l}\text { Aproximación o } \\
\text { distanciamiento }\end{array}$ & $\begin{array}{l}\text { Reacciones ante la pérdida, ¿el cuidador familiar está más cercano a la persona con EA } \\
\text { o ha decidido distanciarse? }\end{array}$ \\
\hline Opinión frente a la muerte & $\begin{array}{l}\text { ¿El proceso de enfermedad del familiar ha hecho que la persona cambie su manera de } \\
\text { ver la muerte? }\end{array}$ \\
\hline
\end{tabular}

Fuente: elaboración propia.

responder a una entrevista semiestructurada de forma individual, que llevó a cabo un psicólogo entrenado durante los meses de junio y julio del 2018. En la evaluación se invitaba a los participantes a narrar su propia experiencia en el cuidado de familiares con Alzheimer en fase avanzada.

El entrevistador mantuvo en todo momento una actitud abierta, genuina y de aceptación, sin emitir juicios de valor, facilitando así la narrativa y la comprensión de los diversos aspectos de interés que fueran retomados en caso de que se requirieran mayores detalles pertinentes y relevantes para el alcance de los objetivos de investigación propuestos. Todas las entrevistas se realizaron en los domicilios de los cuidadores, en su contexto natural para mayor comodidad y con el fin de que la evaluación no supusiera para ellos tener que dejar a su familiar al cuidado de otra persona. Después de la evaluación, se transcribieron las grabaciones y se procedió a realizar el análisis de los datos.

\section{Diseño de la investigación}

El diseño del presente trabajo es transversal de pase único, en único momento temporal.

\section{Análisis de datos}

Se llevaron a cabo análisis descriptivos de las variables sociodemográficas y médicas de interés. Asimismo, para analizar cada una de las entrevistas de forma cualitativa se utilizó el análisis del contenido. Para ello, se formaron agrupaciones y se construyeron categorías que deben ser: pertinentes, exhaustivas y homogéneas. Tras esto, se realizó una codificación identificándose los tópicos más relevantes, y por último, se cuantificó la información. Esta construcción de grupos relacionados hizo más sencillo el análisis y facilitó el estudio del concepto (Fernández, 2002).

En el presente estudio, se empezó con un proceso de codificación que consistió en etiquetar 
y segregar partes de la entrevista. En este caso, contamos con siete dimensiones generales, de las cuales se buscaba en el texto las palabras claves, frases o expresiones que nos pudieran dar a entender que la persona estaba refiriéndose a alguna de estas dimensiones. Hay que tener en cuenta que un mismo fragmento puede estar incluido en más de una categoría, por lo tanto, la búsqueda se dio tanto vertical como transversalmente.

Después de señalar mediante las etiquetas los fragmentos, se extrajeron del texto y fueron reagrupados en un escrito nuevo, el cual nos permitió reconocer parecidos y diferencias. Este proceso de etiquetamiento-disgregación-reagregación se vio impulsado por una tarea de interpretación muy reflexiva. De acuerdo con los objetivos de la investigación, se debe tomar en consideración hasta qué punto conviene señalar las diferencias o ignorarlas (Sayago, 2014). Para realizar un análisis de este tipo es suficiente con construir los datos, procesarlos e interpretarlos. En el presente estudio se utilizó la triangulación mediante la participación de dos investigadoras independientes, una de ellas, psicóloga clínica, y la otra, trabajadora social. De esta manera se buscaba reducir los sesgos, por ello, los análisis presentados son el consenso de ambas investigadoras.

Con el objetivo de conocer las características de la muestra, se llevaron a cabo estadísticos descriptivos de las variables de interés.

\section{Resultados}

\section{Análisis descriptivos}

\section{Variables sociodemográficas y médicas}

Los cuidadores familiares tenían entre 25 y 73 años $(M=52,10 ; D T=15,38)$, de los cuales se asignaron el $50 \%$ al grupo de jóvenes (menores de 55 años) y el resto al de mayores. El $60 \%$ de los cuidadores familiares no tenían convivencia directa con el paciente con EA, sin embargo, todos consideraron que tenían un vínculo fuerte con el familiar que cuidaban.

Tabla 2.

Estadísticos descriptivos de variables sociodemográficas

\begin{tabular}{|c|c|c|}
\hline Variable & Categoría & $\%$ \\
\hline \multirow{3}{*}{ Nivel académico } & Elementales & 40 \\
\hline & Medios & 20 \\
\hline & Superiores & 40 \\
\hline \multirow{3}{*}{ Ocupación } & Activo & 70 \\
\hline & Desempleado & 10 \\
\hline & Jubilado & 20 \\
\hline \multirow{3}{*}{ Niveles socioeconómicos } & Alto & 30 \\
\hline & Medio & 60 \\
\hline & Bajo & 10 \\
\hline \multirow{3}{*}{ Creencias religiosas } & Creyente practicante & 30 \\
\hline & Creyente no practicante & 40 \\
\hline & No creyente, no practicante & 30 \\
\hline
\end{tabular}

Fuente: elaboración propia. 
Un 30\% de la muestra eran cuidadores principales del familiar con EA. Además, un $20 \%$ tenían cargos familiares añadidos, pero el $70 \%$ disponía de apoyo social. Se pueden consultar más estadísticos descriptivos en la tabla 2.

En referencia a las personas con EA, tenían edades comprendidas entre 56-90 años ( $M=75,40$; $D T=4,05)$, de ellos un $10 \%$ sufría EA temprano, el resto tardío. El $70 \%$ se encontraban en fase avanzada, el resto en moderada.

\section{Duelo anticipado}

Dando respuesta al objetivo principal de la investigación de estudiar el desarrollo del duelo anticipado en cuidadores familiares de personas con EA, encontramos que en referencia a los sentimientos y emociones presentados por los cuidadores ante el diagnóstico y durante el proceso de enfermedad de su familiar con EA, todos habían experimentado tristeza y nadie alegría o asco. Más concretamente (figura 1):

Por otro lado, un $90 \%$ reconocieron que era posible que su familiar estuviera al final de la vida. Ejemplos de lo anterior los encontramos en fragmentos como: "Estoy con ella porque sé que habrá un momento que me faltará", "Habrá un día en que no estará y yo quiero aprovechar todo el tiempo posible", "La persona a la que quieres desaparece" o "Sé que no hay nada a hacer".
A pesar de lo anterior, un 30\% tenía esperanza en que la salud de su familiar mejorara o incluso que se curara, pero el $70 \%$ había perdido la esperanza ("No creo que aparezca nada que le cure"). Por ello, un $70 \%$ pensaba que facilitaría el proceso de muerte ("No alargaría artificialmente la vida de mi marido") y un $30 \%$ que no. Por otra parte, todos ellos informaron haberse reorganizado a nivel familiar. Esto podía observarse en oraciones como: "Ha cambiado la forma de relación", "Pienso en cómo era todo antes, ahora es muy diferente" y "Ahora ya no es igual". Además, todos señalaron que sus relaciones habían empeorado ("La forma de relacionarse ha ido a peor porque él va a peor", "Antes de la enfermedad podíamos hacer muchas cosas juntos y ahora no podemos", "Ha pasado de ser la figura paterna, el fuerte de la familia a ser quien más ayuda necesita").

En cuanto al distanciamiento o aproximación, un $80 \%$ de los participantes se acercaron más a su familiar (un 20\% se distanciaron) ("Paso más tiempo con ella", "Me noto más próxima, quiero que esté bueno", "Intento pasar mucho de tiempo con él"; "Nos hemos distanciado", "Creo que me he separado más de él", "Me siento más unida a ella que nunca", "Ya no la siento próxima a mí"). Por último, en relación con el cambio de opinión frente a la muerte, se observó que un $50 \%$ no habían cambiado su perspectiva, y los que lo hicieron $(50 \%)$, fue de forma positiva ("No diría que esta

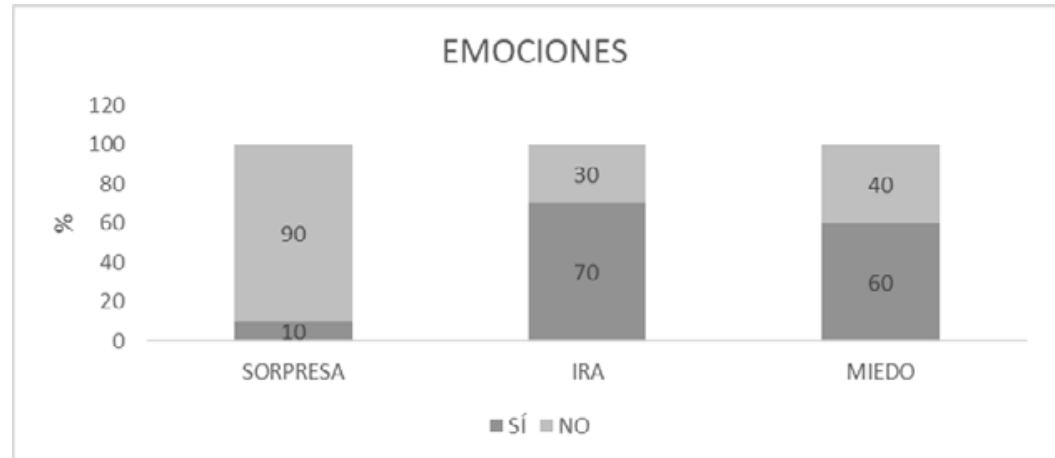

Figura 1. Emociones experimentadas por el cuidador familiar

Fuente: elaboración propia. 
enfermedad ha cambiado la forma de ver la muerte", "Yo siempre he pensado igual, nos puede tocar a todos", "Que mi padre tenga esta enfermedad ha hecho que pasara de no pensar nunca en la muerte a tenerla siempre presente").

\section{Sexo y grupo de edad del entrevistado}

Observamos cómo la totalidad de la muestra ha tenido sentimientos en relación con la pérdida de su ser querido. Concretamente, todos han mostrado tristeza y ninguno felicidad, además todos se ha reorganizado a nivel familiar.

Por otra parte, se observó que todos los hombres manifestaron que han sufrido miedo frente a la situación de final de la vida, y en contraposi- ción, menos de la mitad de las mujeres expresaron que lo han sufrido. Además, todos los hombres informaron de un cambio de opinión acerca de la muerte, a diferencia de una pequeña proporción de mujeres (tabla 3 ).

Con relación a los datos en función de los grupos de edad, encontramos que independientemente del grupo de edad mostraron sentimientos, todos ellos manifestaron tristeza, ninguno felicidad ni asco. Así mismo, se puede observar que el aspecto más diferenciado es la frecuencia de aparición de la esperanza en el cuidado del familiar con EA. En este sentido, es mayor en las personas de más edad. Además, se ve que los jóvenes frente a la situación de final de la vida, viven la muerte de su familiar más positivamente (tabla 3 ).

Tabla 3.

Frecuencias en función del sexo y del grupo de edad del entrevistado

\begin{tabular}{|c|c|c|c|c|c|c|c|c|}
\hline & \multicolumn{2}{|c|}{ Hombre } & \multicolumn{2}{|c|}{ Mujer } & \multicolumn{2}{|c|}{ Joven } & \multicolumn{2}{|c|}{ Adulto y adulto mayor } \\
\hline & Sí & No & Sí & No & Sí & No & Sí & No \\
\hline Sentimientos & 100 & 0 & 100 & 0 & 100 & 0 & 100 & 0 \\
\hline Tristeza & 100 & 0 & 100 & 0 & 100 & 0 & 100 & 0 \\
\hline Felicidad & 0 & 100 & 0 & 100 & 0 & 100 & 0 & 100 \\
\hline Sorpresa & 0 & 100 & 14.30 & 85.70 & 100 & 0 & 20 & 80 \\
\hline Ira & 100 & 0 & 57.10 & 42.90 & 80 & 20 & 60 & 40 \\
\hline Miedo & 100 & 0 & 42.90 & 57.10 & 40 & 60 & 80 & 20 \\
\hline Asco & 0 & 100 & 0 & 100 & 0 & 100 & 0 & 100 \\
\hline Reconocimiento & 100 & 0 & 85.70 & 14.30 & 100 & 0 & 80 & 20 \\
\hline Reorganización & 100 & 0 & 100 & 0 & 100 & 0 & 100 & 0 \\
\hline Esperanza & 0 & 100 & 42.90 & 57.10 & 0 & 100 & 60 & 40 \\
\hline Facilitación & 100 & 0 & 57.10 & 42.90 & 80 & 20 & 60 & 40 \\
\hline Resistencia & 33.30 & 66.70 & 57.10 & 42.90 & 40 & 60 & 60 & 40 \\
\hline Aproximación & & & & & 80 & 20 & 100 & 0 \\
\hline Distanciamiento & 0 & 100 & 28.60 & 71.40 & 40 & 60 & 0 & 100 \\
\hline Opinión & 100 & 0 & 28.60 & 71.40 & 80 & 20 & 20 & 80 \\
\hline
\end{tabular}

Fuente: elaboración propia. 


\section{Situación laboral y académica del entrevistado}

En primer lugar, encontramos que es más frecuente para las personas que se encuentran en activo sorprenderse ante la situación de su familiar. Además, parece más recurrente la ira y la resistencia a la muerte en las personas jubiladas y en el paro, que en las personas en activo (tabla 4).

En relación con los estudios se observó que el grupo que tenía estudios elementales informaba más esperanza que las personas de los grupos con un nivel de estudios más elevados. Este grupo también mostró más resistencia hacia la muerte y no modificó su opinión frente a la situación de final de la vida. Además, fue el grupo que expresó sentir más miedo (tabla 5).

\section{Espiritualidad de los entrevistados}

Se observó que ninguna persona no creyente-no practicante mostraba esperanza en la recuperación, frente al $42.90 \%$ de las personas creyentes. Las personas creyentes se aproximaron emocionalmente más a sus familiares con EA que las que no eran creyentes (tabla 6).

\section{Situación de cuidado de los entrevistados y convivencia con la persona con $\mathrm{EA}$}

Aunque en la mayoría de las situaciones valoradas, las personas que convivían con la persona con EA y las que no convivían mostraban similares valores en los sentimientos ante la situación y en las

Tabla 4.

Frecuencias en función de la situación laboral

\begin{tabular}{|c|c|c|c|c|c|c|}
\hline & \multicolumn{2}{|c|}{ Activo } & \multicolumn{2}{|c|}{ En paro } & \multicolumn{2}{|c|}{ Jubilado } \\
\hline & Sí & No & Sí & No & Sí & No \\
\hline Sentimientos & 100 & 0 & 100 & 0 & 100 & 0 \\
\hline Tristeza & 100 & 0 & 100 & 0 & 100 & 0 \\
\hline Felicidad & 0 & 100 & 0 & 100 & 0 & 100 \\
\hline Sorpresa & 0 & 100 & 0 & 100 & 100 & 0 \\
\hline Ira & 14.3 & 85.7 & 50 & 50 & 0 & 100 \\
\hline Miedo & 57.1 & 42.9 & 100 & 0 & 100 & 0 \\
\hline Asco & 0 & 100 & 0 & 100 & 0 & 100 \\
\hline Reconocimiento de muerte & 85.70 & 14.30 & 100 & 0 & 100 & 0 \\
\hline Reorganización & 100 & 0 & 100 & 0 & 100 & 0 \\
\hline Esperanza & 14.30 & 85.70 & 50 & 50 & 100 & 0 \\
\hline Facilitación & 71.40 & 28.60 & 100 & 0 & 0 & 100 \\
\hline Resistencia & 28.60 & 71.40 & 100 & 0 & 100 & 0 \\
\hline Aproximación & 100 & 0 & 100 & 0 & 100 & 0 \\
\hline Distanciamiento & 28.60 & 71.40 & 0 & 100 & 0 & 100 \\
\hline Opinión & 0 & 100 & 50 & 50 & 57.10 & 42.90 \\
\hline
\end{tabular}

Fuente: elaboración propia. 
características de duelo anticipado, en el caso de las personas que sí eran cuidadores principales se observó que una gran proporción cambió su opinión de forma positiva frente a la muerte, a diferencia de quienes no eran cuidadores principales. De la misma manera, el miedo fue más frecuente en los cuidadores que convivían con las personas con EA, que en aquellos que no lo hacían.

Tabla 5 .

Frecuencia en función del nivel de estudios

\begin{tabular}{|c|c|c|c|c|c|c|}
\hline & \multicolumn{2}{|c|}{ Elementales } & \multicolumn{2}{|c|}{ Medios } & \multicolumn{2}{|c|}{ Superiores } \\
\hline & Sí & No & Sí & No & Sí & No \\
\hline Sentimientos & 100 & 0 & 0 & 100 & 0 & 100 \\
\hline Tristeza & 100 & 0 & 0 & 100 & 0 & 100 \\
\hline Felicidad & 0 & 100 & 0 & 100 & 0 & 100 \\
\hline Sorpresa & 33.30 & 66.70 & 0 & 100 & 0 & 110 \\
\hline Ira & 33.30 & 66.70 & 100 & 0 & 75 & 25 \\
\hline Asco & 0 & 100 & 0 & 100 & 0 & 100 \\
\hline Miedo & 100 & 0 & 66.70 & 33.30 & 25 & 75 \\
\hline Reconocimiento de muerte & 66.70 & 33.30 & 100 & 0 & 100 & 0 \\
\hline Reorganización & 100 & 0 & 0 & 100 & 0 & 100 \\
\hline Esperanza & 100 & 0 & 0 & 100 & 0 & 100 \\
\hline Facilitación & 66.70 & 33.30 & 100 & 0 & 25 & 75 \\
\hline Resistencia & 100 & 0 & 0 & 100 & 50 & 50 \\
\hline Aproximación & 100 & 0 & 100 & 0 & 75 & 25 \\
\hline Distanciamiento & 0 & 100 & 33.30 & 66.70 & 25 & 75 \\
\hline Opinión & 0 & 100 & 100 & 0 & 50 & 50 \\
\hline
\end{tabular}

Fuente: elaboración propia.

Tabla 6.

Frecuencia en función de la espiritualidad de los encuestados

\begin{tabular}{lcccc}
\hline & \multicolumn{2}{c}{ No creyente } & \multicolumn{2}{c}{ Creyente } \\
\hline Sentimientos & Sí & No & Sí No & 0 \\
\hline Tristeza & 100 & 0 & 100 & 0 \\
\hline Felicidad & 100 & 0 & 100 & 0 \\
\hline Sorpresa & 0 & 100 & 100 & 85.70 \\
\hline Ira & 0 & 100 & 71.40 & 28.60
\end{tabular}




\begin{tabular}{lcccc}
\hline & \multicolumn{2}{c}{ No creyente } & \multicolumn{2}{c}{ Creyente } \\
\hline Miedo & Sí & No & Sí & 28.60 \\
\hline Asco & 33.30 & 66.70 & 71.40 & 100 \\
\hline Reconocimiento de muerte & 0 & 100 & 0 & 14.30 \\
\hline Reorganización & 100 & 0 & 85.70 & 0 \\
\hline Esperanza & 100 & 0 & 100 & 57.10 \\
\hline Facilitación & 0 & 100 & 42.90 & 28.60 \\
\hline Resistencia & 66.70 & 33.30 & 71.40 & 57.10 \\
\hline Aproximación & 66.70 & 33.30 & 42.90 & 0 \\
\hline Distanciamiento & 66.70 & 33.30 & 100 & 85.70 \\
\hline Opinión & 33.30 & 66.70 & 14.30 & 57.10 \\
\hline
\end{tabular}

Fuente: elaboración propia.

Tabla 7.

Frecuencias por situación de cuidado y situación de convivencia

\begin{tabular}{|c|c|c|c|c|c|c|c|c|}
\hline & \multicolumn{2}{|c|}{ Cuidador principal } & \multicolumn{2}{|c|}{ Cuidador familiar } & \multicolumn{2}{|c|}{ Convivencia } & \multicolumn{2}{|c|}{ No convivencia } \\
\hline & Sí & No & Sí & No & Sí & No & Sí & No \\
\hline Sentimientos & 100 & 0 & 100 & 0 & 100 & 0 & 100 & 0 \\
\hline Tristeza & 100 & 0 & 100 & 0 & 100 & 0 & 100 & 0 \\
\hline Felicidad & 0 & 100 & 0 & 100 & 0 & 100 & 0 & 100 \\
\hline Sorpresa & 33.30 & 66.70 & 0 & 100 & 33.30 & 66.70 & 0 & 100 \\
\hline Ira & 33.30 & 66.70 & 85.70 & 14.30 & 33.30 & 66.70 & 85.70 & 14.30 \\
\hline Miedo & 66.70 & 33.30 & 57.10 & 42.90 & 100 & 0 & 42.90 & 57.10 \\
\hline Asco & 0 & 100 & 0 & 100 & 0 & 100 & 0 & 100 \\
\hline Reconocimiento & 100 & 0 & 85.70 & 14.30 & 100 & 0 & 85.70 & 14.30 \\
\hline Reorganización & 100 & 0 & 100 & 0 & 100 & 0 & 100 & 0 \\
\hline Esperanza & 66.70 & 33.30 & 14.30 & 85.70 & 66.70 & 33.30 & 14.30 & 85.70 \\
\hline Facilitación & 66.70 & 33.30 & 71.40 & 28.60 & 66.70 & 33.30 & 71.40 & 28.60 \\
\hline Resistencia & 66.70 & 33.30 & 42.90 & 57.10 & 66.70 & 33.30 & 42.90 & 57.10 \\
\hline Aproximación & 100 & 0 & 85.70 & 14.30 & 100 & 0 & 85.70 & 14.30 \\
\hline Distanciamiento & 0 & 100 & 28.60 & 71.40 & 0 & 100 & 28.60 & 71.40 \\
\hline Opinión & 71.40 & 28.60 & 0 & 100 & 33.30 & 66.70 & 57.10 & 42.90 \\
\hline
\end{tabular}

Fuente: elaboración propia. 


\section{Discusión}

Dando respuesta al objetivo planteado de estudiar la presencia de duelo anticipado en familiares de personas con EA y las posibles diferencias de expresión en función de las variables sociodemográficas. Se observó que es bastante frecuente presentar emociones frente a la situación de final de la vida (Soto-Rubio, 2018), lo más habitual es experimentar tristeza, tal y como se mencionó con anterioridad (Parro-Jiménez et al., 2021). Aunque la ira y el miedo también aparecen, la sorpresa no suele estar presente. Ahora bien, observamos cómo el proceso de duelo anticipado no es sinónimo de patología (Strobe et al., 2001) pues aunque surgen emociones, son reacciones normales ante situaciones inesperadas. No obstante, se ha de tener en cuenta que expresar emociones en el proceso de duelo anticipado es saludable, pero la regulación de estas es necesaria para proteger a la persona de desarrollar un duelo patológico (Barreto et al., 2008).

Así mismo, todos los cuidadores familiares se habían reorganizado a nivel familiar para dar respuesta a la nueva situación. Lo anterior va en la línea de otros estudios que señalaban que el duelo anticipado da lugar a un conjunto de tareas adaptativas por parte de los familiares de la persona que va a morir, donde es esencial asumir nuevas responsabilidades, adaptarse al cambio de roles y a la nueva realidad que vive la familia (Lebow, 1976; Palacio \& Bernal, 2017).

La mayoría de los participantes reconocieron que era posible que su familiar estuviera al final de la vida, aspecto esencial del proceso de duelo anticipado (Futterman et al., 1972; Páez-Cala \& Arteaga-Hernández, 2019). Lo anterior puede deberse a que las personas con EA de nuestro estudio se encontraban en fases medias y avanzadas en las cuales el deterioro era grave y visible (AA, 2018), por lo que puede ser más evidente para los cuidadores, la situación de final de la vida.

Aun así, aparecen sentimientos de esperanza ante la muerte; algunos de los cuidadores familiares esperaban que la salud de su familiar mejorara. En esa línea, la literatura no ha llegado a un consenso puesto que algunos autores manifiestan que la esperanza es propia del proceso de duelo anticipado, y otros señalan que durante el proceso de enfermedad crónica la esperanza de que el ser querido se mejore se ha visto destrozada tantas veces, que no suele presentarse en las etapas cercanas a la muerte (Bowlby, 1983; Kübler-Ross, 1998; Páez-Cala \& Arteaga-Hernández, 2019). Por ello, observamos cómo solo algunas personas de nuestra investigación mostraban esperanza.

Los sentimientos de esperanza en nuestra muestra son más frecuentes en personas con menos nivel de estudios. Del mismo modo, estos cuidadores también presentan más resistencia frente a la muerte y no modifican su opinión ante ella. Parece que el nivel de estudios puede ser una variable relevante en la elaboración del duelo anticipado, tal y como sucede en el proceso de duelo tras la muerte (Carreño-Moreno et al., 2019).

Con relación a la facilitación o resistencia ante la muerte, una parte de los participantes (30\%) se resistiría notablemente al hecho de dejar marchar a su familiar. En este sentido, encontramos evidencia muy diversa, puesto que los sujetos mostraron en esta dimensión particularmente mucha disparidad en sus respuestas, mostrando de manera clara el hecho de no tener una formada y firme opinión sobre el tema. Pensamos que esta controversia se debe a la excesiva dureza de la propia dimensión en sí, puesto que es la única dimensión que aún no está presente en la vida de los sujetos. Sin embargo, suele incluirse la facilitación como parte del proceso de aceptación e interiorización de la situación (Bowlby, 1980; Kübler-Ross, 1998; Rando, 2006).

Desde otra perspectiva, encontramos que una pequeña parte de los participantes se distanciaron de su familiar en este momento. Siendo este otro aspecto controvertido en la literatura, puesto que generalmente se ha señalado cierto distanciamiento emocional en estas situaciones (Vega-Vega et al., 
2019). No obstante, hay que tener en cuenta que durante el proceso de duelo hay muchas pérdidas $\mathrm{y}$, por lo tanto, es cierto que puede aparecer cierto desinterés, que no se centra en el individuo en sí, sino en pequeñas cuestiones asociadas a la persona, como los proyectos en común, las costumbres y los hábitos (Gatto, 2006).

La mitad de las personas que participaron en el estudio, cambiaron su opinión ante la muerte de forma positiva. Las personas que modificaban la manera de vivir la muerte lo hacían de forma adaptativa. Otros estudios habían señalado que es frecuente tener opiniones más favorables y positivas frente a la muerte después de convivir con personas cercanas a ella, incorporando las pérdidas como hechos naturales, y, por consiguiente, opinando acerca de la muerte de forma más favorable (Hernández et al., 2017; Loitegui, 2008), observándose en muchas ocasiones un crecimiento post-traumático en estas situaciones.

De manera general, podemos concluir que efectivamente los sujetos que han formado parte de la muestra pueden haber desarrollado duelo anticipado, observándose en ellos gran parte de las características asociadas a este proceso. Ahora bien, la literatura todavía continúa siendo controvertida al respecto y se necesitan estudios que cuenten con más participantes y evaluaciones de manera longitudinal, pues el proceso de duelo es cambiante.

En cuanto al segundo objetivo en el que esperábamos estudiar las variables de presencia de duelo complicado en función de las características sociodemográficas de los cuidadores familiares, observamos principalmente, tal y como ya se había señalado, que existía más riesgo en las personas con menor nivel de estudios (Blanco et al., 2007). Además, encontramos una peor adaptación en la situación de final de la vida en las mujeres, observándose en ellas más esperanza de recuperación frente a la muerte y mayor distanciamiento emocional. Los hombres, por su parte, mostraron más ira y miedo, pero también más facilitación del proceso y un cambio de opinión positiva.
Del mismo modo, las personas mayores de la muestra parecen poseer más indicadores de riesgo, puesto que informan tener más miedo, esperanza y distanciamiento, probablemente porque están más cerca del familiar, siendo la pareja o los hermanos del familiar al final de la vida, por ejemplo. Por su parte, las personas creyentes muestran más esperanza, pero también más aproximación que las personas no creyentes y no practicantes. Lo que podría ir parcialmente en la línea de la literatura, pues se ha señalado la espiritualidad como un factor protector ante el duelo patológico (Barreto et al., 2012). En cuanto a la entrevista solo evaluamos las creencias religiosas, es posible que las personas no creyentes y no practicantes, tuvieran creencias espirituales que fueron tenidas en cuenta.

Aquellos que no convivían con el familiar con EA y los que no eran cuidadores principales muestran más ira frente a la situación. No obstante, los cuidadores principales informan de un cambio de opinión positivo que se acompaña de haber experimentado esperanza a lo largo del proceso de la enfermedad, aspecto que se da más a menudo en los cuidadores que conviven con la persona con EA. Estos últimos también experimentan más miedo que los que no conviven con la persona con EA, debido a que a menudo pueden anticipar la soledad que podrán experimentar una vez la persona muera. La convivencia había sido mencionada en otros estudios como factor de riesgo de desarrollar duelo complicado (Blanco et al., 2007).

Por todo lo anterior, se concluye que podrían existir factores sociodemográficos que facilitan o dificultan el proceso de duelo anticipado, pese a que son necesarios más datos al respeto. Si futuras investigaciones amplían la muestra y combinan la investigación cualitativa con la cuantitativa, podríamos obtener conclusiones más válidas y fiables.

Dicho lo anterior, si se equiparara la muestra por sexos, puede que los resultados fueran distintos, pero tenemos que señalar que nuestro estudio refleja la realidad de los cuidadores familiares, puesto que generalmente suelen ser mujeres 
(Lacomba-Trejo et al., 2017; Martins et al., 2019). Otros estudios podrían tener en cuenta las variables que se han señalado como factores de riesgo en el duelo normativo (Barreto et al., 2012) para comprobar si efectivamente son los mismos o puede ser un proceso totalmente diferenciado.

Así mismo, nuestro estudio puede ser un punto de partida para futuras investigaciones, ya que identifica características de riesgo y protección de los cuidadores familiares. Se debe tener en cuenta a los dolientes que tengan dificultades emocionales, para darles el apoyo asistencial que necesiten (Soto-Rubio et al., 2017). Lo anterior repercutirá en su bienestar pero también en el de la persona a cargo, pues reduciendo el malestar del cuidador podemos disminuir el de la persona a cargo (SotoRubio et al., 2017), en vista de que se ha señalado el estado emocional del cuidador principal como primordial en el ajuste de la persona al final de la vida (Palacios-Espinosa et al., 2015; Soto-Rubio et al., 2018).

Ahora bien, como se ha visto, la mayoría de los estudios se centran en el proceso de duelo normativo (Blanco et al., 2007; Barreto et al., 2008; Soto-Rubio et al., 2018), lo que hace más necesarios los estudios sobre el proceso de duelo anticipado. Nuestros resultados señalan la necesidad de continuar investigando los factores de riesgo y protección en los cuidadores familiares de personas con EA, para poder desarrollar e implementar programas de intervención, teniendo en cuenta la importancia de las variables sociodemográficas y emocionales en la adaptación al duelo anticipado, sabiendo que el duelo anticipado puede ser un proceso todavía más complejo que el duelo tras la muerte (Loitegui, 2008).

Como conclusión, queremos señalar que el duelo anticipado no puede ser considerado ni funcional ni disfuncional para el individuo o su familia (Fulton, 2003), sino que debe ser visto como parte de un proceso normativo e individual que pueden experimentar los individuos cercanos a una persona al final de la vida.

\section{Referencias}

Aranda, M., \& Calabria, A. (2019). Impacto económico-social de la enfermedad de Alzheimer. Neurología Argentina, 11(1), 19-26. https://doi. org/1010.1016/j.neuarg.2018.11.001

Alzheimer's Association. (2018). Alzheimer's disease facts and figures. Alzheimer's \& Dementia, 10(2), e47-e92.

Barreto, P., de la Torre, O., \& Pérez-Marín, M. (2012). Detección de duelo complicado. Psicooncología, 9(2-3), 355-368. https://doi. org/10.5209/rev_PSIC.2013.v9.n2-3.40902

Barreto, P., Yi, P., \& Soler, C. (2008). Predictores de duelo complicado. Psicooncología, 5(2-3), 383-400. http://hdl.handle.net/10550/2135

Blanco, L., Librada, S., Rocafort, J., Cabo, R., Galea, T., \& Alonso, M.E. (2007). Perfil del cuidador principal del enfermo en situación terminal y análisis del riesgo de desarrollar duelo patológico. Medicina Paliativa, 14(3), 10-13. https://acortar. link/BuTVcm

Bowlby, J. (1983). La pérdida afectiva: tristeza y depresión. Ediciones Paidós.

Carreño-Moreno, S., Chaparro-Díaz, L., Carrillo, G. M., \& Gómez-Ramírez, O. J. (2019). Duelo en el cuidador del niño fallecido por cáncer: revisión exploratoria. Duazary, 16(2), 281-294. https:// doi.org/10.21676/2389783X.2755

Casal, B., Rivera, B., \& Currais, L. (2019). Enfermedad de Alzheimer y calidad de vida del cuidador informal. Revista Española de Geriatría y Gerontología, 54(2), 81-87. https://doi. org/10.1016/j.regg.2018.10.008

Castillo, C., Díaz, G., Jelvez, E., \& López, A. (2019). Estrategias de afrontamiento del terapeuta ocupacional en cuidados paliativos infanto-juveniles. Revista de Estudiantes de Terapia Ocupacional, 6(1), 13-21. http://reto.ubo.cl/index.php/reto/ article/view/79

Cohen, J. (1988). Statistical Power Analysis for the Behavioral Sciencies (2nd ed.). Erlbaum. 
Crespo, M., Hornillos, C., \& Gómez, M. M. (2013). Assessing quality of life of nursing home residents with dementia: Feasibility and limitations in patients with severe cognitive impairment. International Psychogeriatrics, 25(10), 1687-1695. https://doi.org/10.1017/S1041610213000823

Fernández, F. (2002). El análisis de contenido como ayuda metodológica para la investigación. Revista de Ciencias Sociales, 96(2), 35-53. https://www.redalyc.org/pdf/153/15309604. pdf

Freud, S. (1917). Mourning and melancholia. The Standard Edition of the Complete Psychological Works of Sigmund Freud, 14, 237-260.

Fulton, G. (2003). Anticipatory mourning: A critique of the concept. Mortality, 8(4), 342-351. https:// doi.org/10.1080/13576270310001613392

Futterman, E. H., Hoffman, I., \& Sabshin, M. (1972). Parental anticipatory mourning. En B. Schoenberg, A.C. Carr, D. Peretz \& A.H. Kutscher (Eds.), Psychosocial Aspects of Terminal Care (pp. 243-272). Columbia University Press.

Gatto, M. E. (2006). Duelo anticipado y conspiración de silencio [Trabajo presentado en la $1^{\mathrm{a}}$ Jornada de Psicooncología]. Problemas clínicos a partir de la comunicación.

Hernández, E., Llibre, J.D., Bosch, R., \& Zayas, T. (2019). Demencia y factores de riesgo en cuidadores informales. Revista Cubana de $\mathrm{Me}$ dicina General Integral, 34(4), 53-63. http:// scielo.sld.cu/scielo.php?pid=S0864-21252018 $000400007 \&$ script $=$ sci arttext\&tlng $=\mathrm{pt}$

Kübler-Ross, E. (1998). Preguntas y respuestas a la muerte de un ser querido. Ediciones Martínez Roca.

Lacomba-Trejo, L., Casaña-Granell, S., Pérez-Marín, M., \& Montoya-Castilla, I. (2017). Estrés, ansiedad y depresión en cuidadores principales de pacientes pediátricos con Diabetes Mellitus Tipo 1. Calidad de Vida y Salud, 10(1), 10-28. http://hdl.handle.net/10550/65600
Lebow, G.H. (1976). Facilitating adaptation in anticipatory mourning. Social Casework, 57(7), 458-465. https://doi.org/10.1177/ 104438947605700707

Lindemann, E. (1944). Symptomatology and management of acute grief. American Journal of Psychiatry, 101, 141-149. https://doi.org/10.1176/ ajp.101.2.141

Loitegui, A. (2008). Duelo anticipado: Sobre el desarrollo del concepto y la importancia de su estudio y abordaje [Tesis de Grado, Universidad de Belgrano]. Repositorio Universidad de Belgrano. http://repositorio.ub.edu.ar/handle/123456789/4783

Martín-Carrasco, M. (2009). Biomarcadores en la enfermedad de Alzheimer: definición, significación diagnóstica y utilidad clínica. Psicogeriatría, 1(2), 101-114. https://www.viguera. com/sepg/pdf/revista/0102/0102_101_114.pdf

Martins, G., Corrêa, L., Caparrol, A. J. D. S., Santos, P. T. A. D., Brugnera, L. M., \& Gratão, A. C. M. (2019). Sociodemographic and health characteristics of formal and informal caregivers of elderly people with Alzheimer's Disease. Escola Anna Nery, 23(2), e20180327. https://doi. org/10.1590/2177-9465-ean-2018-0327

Meza, E.G., García, S., Torres, A., Castillo, L., Sauri, S., \& Martínez, B. (2008). El proceso de duelo. Un mecanismo humano para el manejo de las pérdidas emocionales. Revista de Especialidades Médico-Quirúrgicas, 13(1), 28-31. https:// www.medigraphic.com/cgi-bin/new/resumen. cgi?IDARTICULO $=30270$

Nelson, P. T., Head, E., Schmitt, F. A., Davis, P. R., Neltner, J. H., Jicha, G. A., ... \& Scheff, S. W. (2011). Alzheimer's disease is not "brain aging": Neuropathological, genetic, and epidemiological human studies. Acta Neuropathologica, 121(5), 571-587. https://doi.org/10.1007/s00401-0110826-y

Organización Mundial de la Salud. (2019). Demencia. Organización Mundial de la Salud. 
Páez-Cala, M.L, \& Arteaga-Hernández, L.F. (2019). Duelo por muerte perinatal. Necesidad de una atención diferencial e integral. Archivos de Medicina (Col), 19(1), 32-45. https://doi. org/10.30554/archmed.19.1.2853.2019

Palacio, M.C., \& Bernal, F. (2017). El duelo por muerte: la intersección entre prácticas culturales, rituales sociales y expresiones emocionales. Tempus Psicológico, 2(1), 89-108. https://doi. org/10.30554/tempuspsi.1.2.2595.2019

Palacios-Espinosa, X., \& González, M.I. (2015). Las representaciones sociales del cáncer y de la quimioterapia en la familia del paciente oncológico. Avances en Psicología Latinoamericana, 33(3), 497-515. https://doi.org/10.12804/ ap133.03.2015.09

Palacios-Espinosa, X., Lizarazo, A. M., Moreno, K. S., \& Ospino, J. D. (2015). El significado de la vida y de la muerte para mujeres con cáncer de mama. Avances en Psicología Latinoamericana, 33(3), 455-479. https://dx.doi. org/10.12804/ ap133.03.2015.07

Parkes, C.M., \& Weiss, R.S. (1983). Recovery form bereavement. Basic Books.

Parro-Jiménez, E., Morán, N., Gesteira, C., Sanz, J., \& Garccía-Vera, M.P. (2021). Complicated grief: A systematic review of prevalence, diagnosis, risk and protective factors in the adult population of Spain. Anales de Psicología, 37(2), https://doi.org/10.6018/analesps.37.2.443271.

Payás, A. (2010). Las tareas del duelo: psicoterapia de duelo desde un modelo integrativorelacional. Ediciones Paidós.

Pérez-Godoy Díaz, E., Baños, M. G., \& Javier, A. (2007). Duelo y muerte en cuidadores familiares y profesionales de enfermos de Alzheimer. Gerokomos, 18(2), 15-18. https://doi.org/10.4321/ S1134-928X2007000200002

Rando, T.A. (2000). Clinical dimensions of anticipatory mourning: Theory and practice in working with the dying, their loved ones and their caregivers. Research Press.
Rodríguez, B. C., Castiñeira, B. R., \& Nunes, L. C. (2019). Enfermedad de Alzheimer y calidad de vida del cuidador informal. Revista Española de Geriatría y Gerontología, 54(2), 81-87. https:// doi.org/10.1016/j.regg.2018.10.008

Ruiz-Fernández, M.D., Ortiz, R., \& Ortega-Galán, A. (2019). Estudio cualitativo sobre las vivencias del cuidador de Alzheimer: adaptación e impacto. Cultura de los Cuidados, 23(53). http:// dx.doi.org/10.14198/cuid.2019.53.03

Sayago, S. (2014). El análisis del discurso como técnica de investigación cualitativa y cuantitativa en las ciencias sociales. Cinta de Moebio, (49), 1-10. https://doi.org/10.4067/ S0717-554X2014000100001

Soto-Rubio, A., Pérez-Marín, M., \& Barreto, P. (2017). Frail elderly with and without $\operatorname{cog}$ nitive impairment at the end of life: Their emotional state and the wellbeing of their family caregivers. Archives of Gerontology and Geriatrics, 73, 113-119. https://doi. org/10.1016/j.archger.2017.07.024

Soto-Rubio, A., Pérez-Marin, M., Tomas Miguel, J., \& Barreto Martin, P. (2018). Emotional Distress of Patients at End-of-Life and Their Caregivers: Interrelation and Predictors. Frontiers in Psychology, 9, 2199. https://doi.org/10.3389/ fpsyg.2018.02199

Soto-Rubio, A., Savall, F., Casaña-Granell, S., Molina, P., Giner, J., Zorio, E., ... \& Barreto, P. (2017). Psychosocial and adaptation factors in relatives of patients who died of sudden cardiac death. CorSalud, 9(3), 169-173. https:// www.medigraphic.com/cgi-bin/new/resumenI. cgi?IDARTICULO $=79842$

Soto-Rubio, A. (2018). Beneficios de un programa de soporte para familiares de pacientes al final de la vida: estudio multicéntrico [Tesis doctoral, Universidad de Valencia]. Repositorio Universidad de Valencia. http://roderic.uv.es/ bitstream/handle/10550/66969/TESIS\%20 DOCTORAL\%20A.SOTO\%20\%281\%29. pdf? sequence $=1 \&$ isAllowed $=y$ 
Stroebe, M.S., Hansson, R.O., Stroebe, W., \& Schut, H. (2001). Future directions for bereavement research. En M.S. Stroebe, R.O. Hansson, W. Stroebe \& H. Schut (Eds.), Handbook of bereavement research: Consequences, coping and care (pp. 741-766). American Psychological Association.

Vázquez, A. M. R., \& Campo, M. V. (2019). Vivencias y sentimientos de las personas cuidadoras principales de familiares con enfermedad de Alzheimer. Metas de Enfermería, 22(2), 5-11. https://dialnet.unirioja.es/servlet/articulo?codigo $=6829549$

Vega-Vega, P., González-Rodríguez, R., López-Encina, M. E., Abarca-González, E., Carrasco-Aldunate, P., Rojo-Suárez, L., \& González-Briones, X. (2019). Percepción de apoyo en duelo por profesionales y técnicos de cuidados intensivos pediátricos de hospitales públicos. Revista Chilena de Pediatría, 90(4), 429-436. https:// doi.org/10.32641/rchped.v90i4.1010

Vilajoana, J. (2017). Duelo anticipado en familiares cuidadores de Alzheimer y otras demencias [Tesis doctoral, Universitat de Barcelona]. Repositorio Universitat de Barcelona. https:// www.tdx.cat/bitstream/handle/10803/461798/ JVC_TESIS.pdf? sequence $=1 \&$ isAllowed $=y$
Viñas-Diez, V., Conde-Sala, J. L., Turró-Garriga, O., Gascón-Bayarri, J., Reñé-Ramírez, R., de Demencias, R., \& Conde, J. L. (2019). Síntomas depresivos y sobrecarga en los familiares cuidadores en la enfermedad de Alzheimer: un modelo de ecuaciones estructurales. Revista de Neurología, 67(1), 11-17. https://doi. org/10.33588/rn.6901.2018504

Wilkinson, A. M., \& Lynn, J. (2005). Caregiving for Advanced chronic illness patients. Techniques in Regional Anesthesia and Pain Management, 9, 122-132. https://doi.org/10.1053/j. trap.2005.06.004

Yi, P. Y. (2016). Duelo factores de riesgo de duelo complicado en cuidados paliativos [Tesis doctoral, Universitat de València]. Repositorio Universitat de València. https://core.ac.uk/ download/pdf/71051366.pdf

Zepeda-Álvarez, P.J., \& Muñoz-Mendoza, C.L. (2019). Sobrecarga en cuidadores principales de adultos mayores con dependencia severa en atención primaria de salud. Gerokomos, 30(1), 2-5. https://scielo.isciii.es/scielo.php?pid=S 1134-928X2019000100002\&script $=$ sci_arttext\&tlng=en

\section{Recibido: febrero 25, 2020 Aprobado: septiembre 3, 2021}

